\title{
On the Asymptotic Stability of Reactors with Arbitrary Feedback
}

\author{
A. Z. Akcasu and P. AKhtaR \\ Department of Nuclear Engineering, The University of Michigan, Ann Arbor, Michigan
}

(Received 26 April 1969)

\begin{abstract}
A theorem for the boundedness and asymptotic stability of a point reactor with an arbitrary feedback is stated and proved. The criteria obtained are shown to be essentially the same as those given by Akcasu and Dolfes. The theorem is applied to a reactor with an arbitrary linear feedback and to a xenoncontrolled reactor with a flux reactivity coefficient whose feedback mechanism involves quadratic nonlinearity. It is also compared to a criterion obtained by Corduneanu in the case when delayed neutrons are ignored and the feedback mechanism is linear.
\end{abstract}

\section{INTRODUCTION}

Point kinetic equations for a nuclear reactor with an arbitrary feedback can be written as

$$
\begin{gathered}
\dot{z}=(1+z) \delta K_{f}[z, t]-\sum_{i=1}^{6} a_{i}\left(z-z_{i}\right), \\
\dot{z}_{i}=h_{i}\left(z-z_{i}\right), \quad i=1,2, \cdots, 6, t \geq 0,
\end{gathered}
$$

where $t$ denotes time and the dot indicates the first derivative of the dependent variables $z(t)$ and $z_{i}(t)$ with respect to $t$. The functions $z(t)$ and $z_{i}(t)$ represent the incremental reactor power and delayed neutron precursor densities, respectively. ${ }^{1}$ The parameters $a_{i}$ and $h_{i}$ are positive numbers, with the $a_{i}$ satisfying the relation

$$
\sum_{i=1}^{6} a_{i}=1 .
$$

The symbol $\delta K_{f}[z, t]$ denotes a functional of the function $z(t)$ involving values of the latter in the interval $(-\infty, t)$. We shall always assume that $\delta K_{f}[z, t]$ can be represented as

$$
\begin{aligned}
& \delta K_{f}[z, t] \\
& =\int_{-\infty}^{t} d u G_{1}(t-u) z(u) \\
& \quad+\int_{-\infty}^{t} d u \int_{-\infty}^{t} d v G_{2}(t-u, t-v) z(u) z(v)+\cdots,
\end{aligned}
$$

where $G_{1}(u), G_{2}(u, v)$, etc., are linear, quadratic, etc., feedback kernels. These kernels are defined only for positive arguments, but it turns out to be convenient

\footnotetext{
1. The usual point reactor kinetic equations ${ }^{2}$ can be reduced to the form of Eqs. (1) and (2) by choosing $z(t)=\left[P(t)-P_{0}\right] / P_{0}$, $z_{i}(t)=\left[C_{i}(t)-C_{i 0}\right] / C_{i 0}$, and by letting $\rightarrow \beta t / l, a_{i}=\beta_{i} / \beta$, and $h_{i}=l \lambda_{i} / \beta . P_{0}$ and $C_{i 0}$ denote equilibrium values of reactor power and delayed neutron precursor densities, respectively, and $l, \lambda_{i}, \beta_{i}$, $\beta$ have their conventional meanings in the field of reactor engineering. $P_{0}$ and $C_{i 0}$ are determined by $K_{0}+K_{f}\left(P_{0}\right)=0$ and $C_{i 0}=a_{i} P_{0} / \lambda_{i}$, where $K_{0}$ and $K_{f}\left(P_{0}\right)$ are external and equilibrium feedback reactivities. The incremental feedback reactivity functional $\delta K_{f}[z, t]$ appearing in Eq. (1) is then defined as $\delta K_{f}[z, t] \equiv K_{f}[P, t]-K_{f}\left(P_{0}\right)$, where $K_{f}[P, t]$ is the total feedback reactivity functional. We will assume that the algebraic equation relating $K_{0}$ and $K_{f}\left(P_{0}\right)$ has a unique solution. We also note that $(1+z)$ and $\left(1+z_{i}\right)$ are nonnegative, since $P_{0}$ and $C_{i 0}$ can never be negative.

A. F. Henry, Nucl. Sci. Eng. 3, 52 (1958).
}

to define them to be identically zero for negative arguments.

Physically, $\delta K_{f}[z, t]$ denotes the feedback reactivity at $t$ due to the power generation in the reactor prior to $t$. From the physical nature of the feedback mechanism we require $\delta K_{f}[z, t]$ to be a bounded function of time whenever $z(t)$ is bounded (stability of the feedback). Mathematically this condition is satisfied if we assume that the kernels $G_{n}\left(u_{1}, u_{2}, \cdots\right.$, $u_{n}$ ) are absolute integrable, i.e.,

$$
\gamma_{n}=\int_{0}^{\infty} d u_{1} \cdots \int_{0}^{\infty} d u_{n}\left|G_{n}\left(u_{1}, u_{2}, \cdots, u_{n}\right)\right|
$$

and that the power series $\sum_{n-1}^{\infty} \gamma_{n} M^{n}$ is convergent for all finite values of $M$ where $M$ is the bound of $z(t)$.

Equations (1) and (2) describe the temporal behavior of $z(t), z_{i}(t)$ only for positive $t$. Since the feedback mechanism depends on the values of $z(t)$ in the interval $(-\infty, 0)$ as well as in $(0, t)$, a unique solution of this set of equations requires a specification not only of the initial value $z(0)$ and $z_{i}(0)$ but also of the values of $z(t)$ prior to $t=0$. We shall refer to $z(t)$ for $t \leq 0$ as an initial curve. It is clear from the form of Eqs. (1) and (2) and the definition of $\delta K_{f}[z, t]$ that $z(t)=z_{i}(t) \equiv 0, t>0$, is a solution of Eqs. (1) and (2) corresponding to the initial curve $z(t) \equiv 0$ for $t \leq 0$. We shall refer to this solution as the equilibrium state. $^{3}$ A nonzero initial curve, which is the response of the reactor to external reactivity changes and external sources for $t \leq 0$, can be regarded as a perturbation on this equilibrium state.

The question of stability involves the behavior of $z(t)$ and $z_{i}(t)$ for $t>0$, and in particular when $t \rightarrow \infty$. Since these functions depend on the nature of the initial curves as well as the initial values, one

$z(t)=z_{i}(t) \equiv-1$ for all $t$ also represents an equilibrium state. Physically, this corresponds to a reactor in which there are no neutrons and delayed neutron precursors. In the course of a derivation we shall exclude this equilibrium state from discussion because it will be shown that, when conditions for boundedness as stated in the theorem in the next section are satisfied, $z(t)$ and $z_{i}(t)$ can never approach -1 [cf. Eqs. (14a) and (14b)] once the reactor is perturbed. 
must first specify a certain class of initial curves in order to state conditions for stability. This class must be sufficiently broad to include all the possible perturbations that may arise intentionally or accidentally during the operation of the reactor. On physical grounds we choose this class of initial curves, henceforth called physically admissible initial curves, to satisfy the following properties:

(i) $z(t) \equiv 0$ for $t \leq-t_{0}, t_{0}>0$;

(ii) $z(t)>-1,-t_{0} \leq t \leq 0$;

(iii) $z(t)$ is piecewise continuous and bounded, and its first derivative exists and is bounded at all times, from both left- and right-hand side, in the interval $\left(-t_{0}, 0\right)$.

Condition (i) implies that perturbations are confined to a finite time interval. Condition (ii) follows from the positivity of reactor power during reactor operation. The boundedness and piecewise continuity of $z(t)$ and the boundedness of its first derivative are indicated by the fact that an initial curve is the response of a reactor to an external disturbance either in the reactivity or in the source for $t \leq 0$. Jump discontinuities are permitted to allow for the presence of a possible pulse source in the time interval $\left(-t_{0}, 0\right)$ :

We shall not reproduce here the various precise definitions of stability. ${ }^{4}$ We shall be mainly concerned with asymptotic stability in the large which we define, for any physically admissible initial curve and for finite $z(0)$ and $z_{i}(0)$, as

$$
\lim _{t \rightarrow \infty} z(t)=0 .
$$

A criterion for the asymptotic stability of the reactors described by Eqs. (1) and (2) was derived by Akcasu and Dalfes ${ }^{5}$ with an heuristic approach. The derivation was largely based on intuitive reasoning and the results were justified on the basis of energy considerations. In the present work a rigorous analysis is carried out and the results are stated in the form of a theorem. It is found that in addition to the criterion in Ref. 5 certain additional restrictions have to be imposed on the feedback functional $\delta K_{f}[z, t]$ to guarantee asymptotic stability. These restrictions, however, turn out to be not very stringent and seem to be quite compatible with physically realizable reactor systems. This will be demonstrated for reactors with linear feedback and for a xenoncontrolled reactor with flux reactivity coefficient

\footnotetext{
N. N. Krasovskii, Stability of Motion (Stanford University Press, Stanford, California, 1963).

S A. Z. Akcasu and A. Dalfes, Nucl. Sci. Eng. 8, 89 (1960).
}

where the feedback functional is nonlinear. ${ }^{6,7}$ In the course of the derivation of the stability criterion, it is also observed that the restrictions on $\delta K_{f}[z, t]$ are connected with conditions imposed on initial curves. The choice of an unrealistically broad class of initial curves for the sake of mathematical generality will yield sufficient conditions for asymptotic stability which may turn out to be too restrictive to be of any practical interest for reactor applications. This necessitates the restriction of the initial perturbations to physically admissible initial curves as defined above.

\section{STABILITY THEOREMS}

Theorem 1 (Boundedness): The response of a nuclear reactor, described by Eqs. (1) and (2), is always bounded for any physically admissible initial curve if:

(i) $z(0), z_{i}(0)$ are bounded and greater than -1 ;

(ii) $\int_{-\infty}^{t} \delta K_{f}\left[y, t^{\prime}\right] y\left(t^{\prime}\right) d t^{\prime} \leq 0, t>0$, for all test functions $\{y(t)\}$ which belong to the class of physically admissible initial curves for $t \leq 0$ and are arbitrary for $t \geq 0 .{ }^{8}$

Theorem 2 (Asymptotic Stability): The response $z(t)$ is asymptotically stable in the large if, in addition to Theorem 1, the feedback functional satisfies the following conditions:

(i) $\delta K_{f}[v, t]$ is uniformly continuous for sufficiently large $t$ for all test functions $\{v(t)\}$ which belong to the class of physically admissible initial curves for $t \leq 0$, and are continuous, bounded, and have bounded first derivatives for $t \geq 0$.

(ii) $\lim \delta K_{f}[\omega, t]=0$, as $t \rightarrow \infty$, implies $\lim \omega(t)=$ 0 , as $t \rightarrow \infty$, for all test functions $\{\omega(t)\}^{9}$ which belong to the class of physically admissible initial curves for $t \leq 0$ and, for $t \geq 0$, are continuous, bounded, and have uniformly continuous first derivatives which vanish as $t \rightarrow \infty$.

\section{THE PROOF OF BOUNDEDNESS}

We consider a functional of $z(t)$ defined as

$$
\begin{array}{r}
V[z, t]=F(z)+\sum_{i=1}^{6} \frac{a_{i}}{h_{i}} F\left(z_{i}\right)-\int_{-\infty}^{t} \delta K_{f}\left[z, t^{\prime}\right] z\left(t^{\prime}\right) d t^{\prime}, \\
t \geq 0, \quad(7)
\end{array}
$$

${ }^{6}$ J. Chernick, G. Lellouche, and W. Wollman, Nucl. Sci. Eng. $10,120(1960)$.

A. Z. Akcasu and P. Akhtar, J. Nucl. Energy 21, 341 (1967).

${ }^{8}$ It should be noted that there is no restriction on the bound of the test functions $\{y(t)\}$ for $t \geq 0$, and they may diverge as $t \rightarrow \infty$. Clearly $\{y(t)\}$ contains all possible solutions of Eqs. (1) and (2) as a subset.

${ }^{9}$ It may be noted that the test functions $\{\omega(t)\}$ form a subset of the functions $\{v(t)\}$, which in turn are a subset of test functions $\{y(t)\}$. 
in which $F(u), u=z, z_{1}, \cdots, z_{6}$, is defined as

$$
F(u) \equiv u-\ln (1+u) \equiv \int_{0}^{u} \frac{x}{1+x} d x, \quad u>-1 .
$$

Variations of $F(u)$ with $u$ are shown in Fig. 1. It is a positive definite function possessing the following properties: $u=0$

(i) $F(u)$ is real and positive in $(-1, \infty)$ except for

(ii) $F(0)=0$;

(iii) $F(u)$ is continuous in $(-1, \infty)$.

The first two terms of $V[z, t]$ in Eq. (7) are thus nonnegative, since $a_{i}, h_{i}$ are known to be positive constants. The last term is also nonnegative if condition (ii) for boundedness is imposed for all test functions $\{y(t)\}$ and thus necessarily for any possible trajectory $z(t)$ corresponding to a given physically admissible initial curve. Hence,

$$
V[z, t] \geq 0 .
$$

The initial value $V(0)$ is given by

$$
V(0)=F\left(z_{0}\right)+\sum_{i=1}^{6} \frac{a_{i}}{h_{i}} F\left(z_{i 0}\right)-\int_{-\infty}^{0} \delta K_{f}\left[z, t^{\prime}\right] z\left(t^{\prime}\right) d t^{\prime} .
$$

It will be seen that $V(0)$ is finite since initial values $z_{0}$ and $z_{i 0}$ are finite (condition (i)) and the finiteness of the last term,

$$
\int_{-\infty}^{0} \delta K_{f}\left[z, t^{\prime}\right] z\left(t^{\prime}\right) d t^{\prime}
$$

is guaranteed by the finiteness of the physically admissible initial curves and feedback stability. Moreover, by differentiating $V[z, t]$ with respect to $t$ and using Eqs. (1) and (2), we obtain the derivative

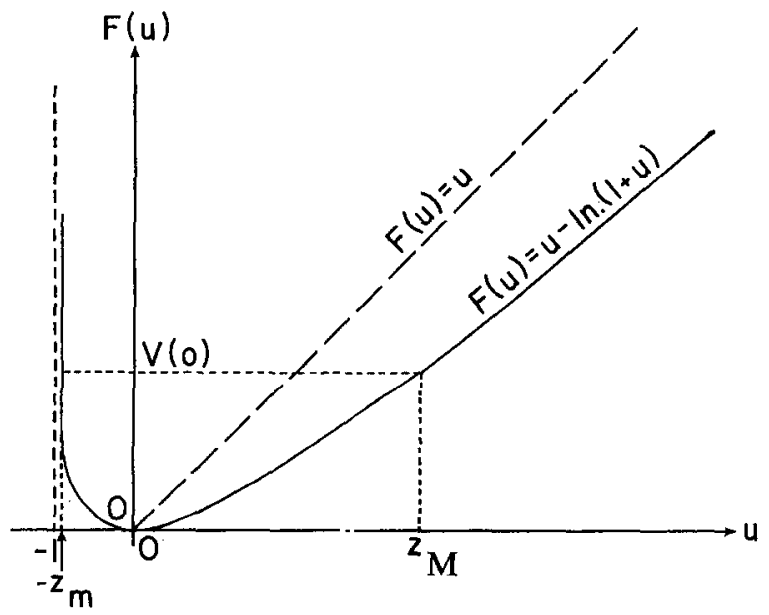

FIG. 1. of $V[z, t]$ along a trajectory as

$$
\dot{V}[z, t]=-\sum_{i=1}^{6} \frac{a_{i}\left(z-z_{i}\right)^{2}}{(1+z)\left(1+z_{i}\right)}, \quad t>0,
$$

which is continuous whenever $z(t) \neq-1$ and $z_{i}(t) \neq$ -1 inasmuch as $z(t)$ and $z_{i}(t)$ are continuous in $t$ by virtue of Eqs. (1) and (2). Furthermore, since $(1+z)$ and $\left(1+z_{i}\right)$ are nonnegative by definition, ${ }^{1}$

$$
\dot{V}[z, t] \leq 0, \quad t>0 ;
$$

the equality occurs when $z=z_{i}$.

It is thus concluded that $V[z, t]$ is a nonnegative, monotonically decreasing function of time along a trajectory. In particular, we obtain

$$
V[z, t] \leq V(0),
$$

which indicates that $V[z, t]$ is finite for all $t \geq 0$ if $V(0)$ is finite. We observe that $V[z, t]$ is a sum of three nonnegative terms (cf. Eq. 7). Therefore, each term in Eq. (7) is smaller than $V(0)$ for all $t \geq 0$ :

$$
\begin{aligned}
& F(z) \leq V(0), \\
& F\left(z_{i}\right) \leq V(0)\left(h_{i} / a_{i}\right), \\
&\left|\int_{-\infty}^{t} \delta K_{f}\left[z, t^{\prime}\right] z\left(t^{\prime}\right) d t^{\prime}\right| \leq V(0) .
\end{aligned}
$$

The inequalities (13a) and (13b), with the help of Fig. 1, imply that

$$
\begin{aligned}
& -1<-z_{m} \leq z(t) \leq z_{M}, \\
& -1<-z_{m_{i}} \leq z_{i}(t) \leq z_{M_{i}},
\end{aligned}
$$

where $z_{m}, z_{M}, z_{m_{i}}$, and $z_{M_{i}}$ are positive numbers such that

and

$$
F\left(z_{M}\right)=F\left(-z_{m}\right)=V(0), \quad z_{M}>z_{m},
$$

$$
F\left(z_{M_{i}}\right)=F\left(-z_{m_{i}}\right)=V(0) h_{i} / a_{i}, \quad z_{M M_{i}}>z_{m_{i}} .
$$

The inequalities (14a) and (14b) establish the boundedness of the reactor response to any physically admissible initial curve. Furthermore, they also prove that both $z(t)$ and $z_{i}(t)$ are bounded away from -1 for $t>0$, and hence the power can never approach the shutdown equilibrium state (cf. Ref. 3 ).

The conditions of Theorem 1, apart from the boundedness of $z(t)$ and $z_{i}(t)$, also lead to the following conclusions which will be useful in proving asymptotic stability:

(i) $\dot{z}(t)$ is bounded for all $t \geq 0$; this follows from Eq. (1) and stability of the feedback; that is,

$$
|\dot{z}(t)| \leq\left(1+z_{M}\right) W+2 z_{M},
$$

where $z_{M}$ is assumed to represent the largest value of the upperbounds $z_{M}, z_{M_{1}}, \cdots, z_{M_{8}}$ and $W$ is the 
upper bound of $\delta K_{f}[z, t]$ corresponding to the given physically admissible initial curve. Hence $z(t)$ is not only bounded but also uniformly continuous.

(ii) $z_{i}(t)$ is also uniformly continuous, since

[cf. Eq. (2)].

$$
\left|\dot{z}_{i}(t)\right| \leq 2 h_{i} z_{M}
$$

(iii) $\dot{V}(t)$ is continuous for all $t \geq 0$ because $(1+z)$ and $\left(1+z_{i}\right)$ are never zero by virtue of Eqs. (14a) and (14b) [cf. Eq. (10)]. Moreover,

and

$$
|\dot{V}(t)| \leq 4 z_{M}^{2} /\left(1-z_{m}\right)^{2}
$$

$$
\begin{aligned}
|\ddot{V}(t)| & \leq \frac{4 z_{M}\left(1+z_{M}\right)}{\left(1-z_{m}\right)^{3}} \\
& \times\left(\left(1+z_{M}\right) W+2 z_{M}\left(1+\sum_{i=1}^{\mathfrak{B}} \operatorname{aihi}\right)\right),
\end{aligned}
$$

where $z_{m}$ is taken to be the least value of the lower bounds $z_{m}, z_{m_{1}}, \cdots, z_{m_{s}}$. Hence $\dot{V}(t)$ is bounded and uniformly continuous for all $t \geq 0$.

\section{THE PROOF OF ASYMPTOTIC STABILITY}

In proving the theorem for asymptotic stability we make use of a lemma by Barbalat ${ }^{10}$ which is reproduced here for convenience.

Lemma: Let $g(t)$ be a real function of a real variable $t$, defined for $t>a>0$. If

(i) $\lim g(t)=g_{\infty}$, as $t \rightarrow \infty$, ( $g_{\infty}$ is finite), and

(ii) $\dot{g}(t)$ is uniformly continuous for $t>a$, then $\lim \dot{g}(t)=0$, as $t \rightarrow \infty$.

We start with the observation that

$$
\lim _{t \rightarrow \infty} V(t)=V_{\infty} \geq 0
$$

because $V[z, t]$ is nonnegative and monotomically decreasing. Also, $\dot{V}(t)$ is uniformly continuous (cf. Eqs. 18a, 18b). The lemma is thus applicable for $g(t)=V(t)$ and we have

$$
\lim _{t \rightarrow \infty} \dot{V}(t)=0
$$

along a trajectory. Combining Eqs. (10) and (20) we conclude that

$$
\lim _{t \rightarrow \infty}\left[z(t)-z_{i}(t)\right]=0, \quad i=1,2, \cdots, 6,
$$

which, by virtue of Eq. (2), leads to

$$
\lim _{t \rightarrow \infty} \dot{z}_{i}(t)=0 \text {. }
$$

We next note that $\dot{z}(t)$ [cf. Eq. (1)] is uniformly continuous for large $t$ since $\delta K_{f}[v, t]$ is uniformly continuous for all test functions $\{v(t)\}$ [cf. condition (i) of the Theorem 2] and thus necessarily for any trajectory $z(t)$. It may be noted that limiting the requirement of uniform continuity of $\delta K_{f}[v, t]$ to only bounded and continuous test functions having bounded derivatives, instead of the set of test functions $\{y,(t)\}$ (which are quite arbitrary and unbounded), is made possible because we already have established the boundedness of $z(t)$ and $\dot{z}(t)$. Moreover, $\dot{z}_{i}(t)$ is uniformly continuous by virtue of Eq. (2) and the fact that $z(t)$ and $z_{i}(t)$ are uniformly continuous as shown above [cf. Eqs. (16), (17)].

The conditions of the lemma are thus satisfied for the function $g \equiv\left[z(t)-z_{i}(t)\right]$ with $g_{\infty}=0$ and hence $\lim \dot{g}=\left[\dot{z}-\dot{z}_{i}\right]=0$, as $t \rightarrow \infty$.

The latter together with Eq. (22) leads to

$$
\lim _{t \rightarrow \infty} \dot{z}(t)=0,
$$

which, in view of Eqs. (1) and (21), and the fact that $z(t)$ cannot approach -1 , leads to

$$
\lim _{t \rightarrow \infty} \delta K_{f}[z, t]=0 .
$$

Condition (ii) can now be applied since we have shown that $z(t)$ is continuous, bounded, and has a uniformly continuous derivative which vanishes as $t \rightarrow \infty$ and thus belongs to the subset $\{\omega(t)\}$. Hence

$$
\lim _{t \rightarrow \infty} z(t)=0
$$

and the equilibrium state $z(t) \equiv 0$ is asymptotically stable.

\section{APPLICATIONS}

In this section we apply the stability theorem to investigate the asymptotic stability of a reactor with an arbitrary linear feedback, and of a xenon-controlled reactor with a flux reactivity coefficient whose feedback mechanism involves quadratic nonlinearity. ${ }^{6,7,11}$

\section{A. Linear Feedback}

The incremental feedback functional in this case reduces to

$$
\delta K_{f}[z, t]=\int_{-\infty}^{t} G(t-u) z(u) d u .
$$

The stability of the feedback mechanism requires the feedback kernel to be absolutely integrable, ${ }^{12}$ i.e.,

$$
\int_{0}^{\infty}|G(u)| d u<\infty
$$

\footnotetext{
${ }^{10}$ I. Barbalat, Rev. Math. Pures Appl. 4, 267 (1959).

11 G. S. Lellouche, J. Nucl. Energy 21, 519 (1967).

12 The condition (26) is necessary and sufficient ${ }^{18}$ for the linear functional $\delta K_{f}[z, t]$ in Eq. (25) to be bound for all $t$ and for all bounded functions $z(t)$ in $(-\infty,+\infty)$.

1s A. Papoulis, Probability, Random Variables, and Stochastic Processes (McGraw-Hill Book Co., New York, 1965).
} 
It can be further proved ${ }^{6}$ that condition (ii) of Theorem 1 , which in this case reduces to

$$
\int_{-\infty}^{t} d t^{\prime} \int_{-\infty}^{t^{\prime}} d t^{\prime \prime} G\left(t^{\prime}-t^{\prime \prime}\right) y\left(t^{\prime}\right) y\left(t^{\prime \prime}\right) \leq 0
$$

is satisfied for all test functions $\{y(t)\}$ if

$$
R_{e}[G(i \omega)] \leq 0
$$

holds $^{7}$ for all $\omega$ in $(0, \infty)$; $\bar{G}(i \omega)$ is the Laplace transform of $G(t)$ with $i \omega$ as the Laplace variable. Hence the reactor response is bounded if the initial perturbation is a physically admissible initial curve, $z(0)$ and $z_{i}(0)$ are bounded, and relations (26) and (28) hold.

For asymptotic stability we first show the uniform continuity of $\delta K_{f}[v, t]$ for large $t$ and for all the functions $\{v(t)\}$ which are continuous, bounded, and have bounded first derivatives for $t \geq 0$.

For $t \leq 0, v(t)$ is a physically admissible initial curve and thus has bounded left and right derivatives, though it might contain jump discontinuities. Let these discontinuities occur at $-T_{1},-T_{2},-T_{3}, \cdots$, $-T_{N}$ with $b_{1}, \cdots, b_{N}$ as respective jumps. Then, by differentiating $\delta \dot{V}_{f}[v, t]$ with respect to $t$, it can be shown that

$$
\left|\delta \dot{K}_{f}[v, t]\right| \leq M \int_{0}^{\infty}|G(t)| d t+\sum_{i=1}^{N}\left|b_{i}\right|\left|G\left(t+T_{i}\right)\right|,
$$

where $M$ is the maximum of $\dot{v}(t)$ for $t$ in the entire domain $\left(-t_{0},+\infty\right)$. The first term in the right-hand side of relation (29) is bounded by virtue of relation (26). The second term vanishes as $t \rightarrow \infty$ as required by relation (26). Hence, $\left|\delta \dot{K}_{f}[v, t]\right|$ is bounded and $\delta V_{f}[v, t]$ is uniformly continuous for sufficiently large times. We note that the continuity of $G(t)$ is not assumed in these discussions; it may have jump discontinuities and it may even contain delta functions in finite time intervals.

Next we have to find conditions that will ensure that

$$
\lim _{t \rightarrow \infty} \int_{0}^{\infty} G(u) \omega(t-u)=0
$$

implies that

$$
\lim _{t \rightarrow \infty} \omega(t)=0
$$

for all test functions $\{\omega(t)\}$ [cf. condition (ii) of Theorem 2]. These conditions immediately follow from Wiener's theorem (Pitts form) ${ }^{14}$ which states that

$$
\lim _{t \rightarrow \infty} \int_{-\infty}^{+\infty} G(t-u) \omega(u) d u=A \int_{-\infty}^{+\infty} G(t) d t
$$

${ }^{14}$ D. V. Widder, The Laplace Transform (Princeton University Press, Princeton, N.J., 1946). implies that

$$
\lim _{t \rightarrow \infty} \omega(t)=A
$$

if:

(i) $G(t)$ is absolute integrable in $(-\infty,+\infty)$ and its Fourier transform $G(i \omega)$ does not vanish anywhere;

(ii) $\omega(t)$ is bounded and has a derivative which remains greater than a negative constant.

The theorem is applicable since Eq. (30a) can be written in the form of Eq. (31a) $[G(u)=0$ for $u \leq 0$ ] and any test function of the subset $\{\omega(t)\}$ is bounded and has bounded first derivatives. We thus obtain the following criterion from Eqs. (30) and (31). If, in addition to relations (26) and (28), $z(0)$ and $z_{i}(0)$ are bounded and we can demonstrate that

$$
\bar{G}(i \omega) \neq 0, \quad-\infty<\omega<+\infty,
$$

then a reactor with linear feedback is asymptotically stable for all physically admissible initial curves. It is clear that Eqs. (28) and (32) will always be satisfied if we require that $R_{e}[G(i \omega)]$ and $I_{m}[G(i \omega)]$ do not vanish at the same frequency. We also note that if the equality sign in Eq. (28) is removed, then $\bar{G}(i \omega)$ cannot vanish at any frequency and Eq. (32) is always satisfied. Hence the condition

$$
R_{\varepsilon}[G(i \omega)]<0
$$

is sufficient for asymptotic stability. This is the wellknown criterion of Welton.

It may be noted that Wiener's theorem does not make explicit use of the fact that in our case $\lim \dot{\omega}(t)$, as $t \rightarrow \infty$, approaches 0 . By exploiting this property, we can replace the condition (32) by a different condition which may be more easily applicable in certain specific cases of the feedback kernel $G(t)$. We first observe that, since $\omega(t)$ is bounded,

$$
\left|\int_{-\infty}^{T} G(t-u)[\omega(u)-\omega(t)] d u\right| \leq 2 M \int_{t-T}^{\infty}|G(u)| d u,
$$

where $T$ is finite and $M$ is the maximum of $\omega(t)$ in $\left(-t_{0}, T\right)$. The right side of relation (33) vanishes as $t \rightarrow \infty$ since $G(u)$ is absolutely integrable. Hence,

$$
\lim _{t \rightarrow \infty} \int_{-\infty}^{T} G(t-u)[\omega(u)-\omega(t)] d u=0 .
$$

Moreover, by expanding $\omega(t)$ as $\omega(t)=\omega(u)+$ $(t-u) \dot{\omega}[u+\theta(u)]$, where $u \leq \theta(u) \leq t$, and choosing $T(\epsilon)$ for a given $\epsilon>0$, no matter how small, such 
that $\dot{\omega}(u)<\epsilon$ for $u>T(\epsilon)$, we have

$$
\begin{aligned}
\mid \int_{T}^{t} G(t-u)[\omega(u)-\omega(t)] & d u \mid \\
& \leq \epsilon \int_{T}^{t}|G(t-u)||t-u| d u \\
& \leq \epsilon \int_{0}^{\infty}|G(u)| u d u
\end{aligned}
$$

Therefore, if we impose the condition that

$$
\int_{0}^{\infty}|G(u)| u d u<\infty,
$$

then it is observed that

$$
\lim _{t \rightarrow \infty} \int_{T}^{t} G(t-u)[\omega(u)-\omega(t)] d u=0
$$

Relations (34) and (37) are sufficient to show that

$$
\lim _{t \rightarrow \infty}\left[\int_{0}^{\infty} G(u) \omega(t-u) d u-\omega(t) \int_{0}^{\infty} G(u) d u\right]=0 .
$$

It can thus be concluded that, if the condition (36) is assumed to hold, then

$$
\int_{0}^{\infty} G(u) \omega(t-u) d u
$$

behaves as $\omega(t)$ for large values of $t$ and, therefore, by virtue of Eq. (30a),

$$
\lim _{t \rightarrow \infty} \omega(t)=0 .
$$

The condition (36) may be observed to be very relaxed. It is, for example, always satisfied when $G(t)$ can be expressed as a sum of exponential terms with negative exponents, such as in the case when the feedback can be described by a set of coupled linear differential equations.

\section{B. Nonlinear Feedback}

In order to illustrate the application of stability theorems to reactors with nonlinear feedback, we consider a xenon-controlled reactor with flux reactivity coefficient for which $\delta K_{f}[z, t]$ is quadratic and has the form ${ }^{7}$

$$
\begin{aligned}
& \delta K_{f}[z, t] \\
& =\int_{-\infty}^{t} d u G_{1}(t-u) z(u) \\
& \quad+\int_{-\infty}^{t} d u_{1} \int_{-\infty}^{t} d u_{2} G_{2}\left(t-u_{1}, t-u_{2}\right) z\left(u_{1}\right) z\left(u_{2}\right),
\end{aligned}
$$

where

$$
\begin{aligned}
G_{1}(t) & =A_{1} \delta(t)+K(t) \\
K(t) & =A_{2} e^{-\lambda_{\alpha} t}+A_{3} e^{-\lambda t}+A_{4} e^{-\lambda_{1} t}
\end{aligned}
$$

and

$$
G_{2}(t)=A_{5} \delta\left(t_{1}-t_{2}\right) e^{-\lambda_{x} t}
$$

$A_{1}, A_{2}, \cdots, A_{5}$ are constants (defined in Ref. 7) depending upon various reactor parameters, and $\lambda_{x}$, $\lambda_{1}, \lambda$ are decay constants of ${ }^{135} \mathrm{Xe},{ }^{135} \mathrm{I}$, and one group delayed neutron precursor concentration, respectively. $G_{1}(t)$ and $G_{2}\left(t_{1}, t_{2}\right)$ may be noted to be absolutely integrable. The reactor response $z(t)$ to any initial perturbation belonging to the class of physically admissible initial curves and for finite $z(0)$ and $z_{i}(0)$ is bounded if

$$
\int_{-\infty}^{t} \delta K_{f}\left[y, t^{\prime}\right] y\left(t^{\prime}\right) d t^{\prime} \leq 0
$$

[condition (ii) of Theorem 1], for all continuous test functions belonging to the set $\{y,(t)\}$. The condition (40), which was derived on the basis of energy considerations in Ref. 5 as a sufficient condition for asymptotic stability, was investigated for combined xenon and temperature feedback [cf. Eq. (39)] in Refs. 7 and 11, and was shown to lead to the following criterion: if the condition

$$
R_{e}[R(i \omega)]+A_{1}-\left(A_{5} / \lambda_{x}\right) P_{0} \leq 0,
$$

where $R(i \omega)$ is the Fourier transform of $K(t)$ [cf. Eq. (39c)] and $P_{0}$ is the equilibrium power level (cf. footnote 1), is satisfied for all real $\omega$, then the reactor is asymptotically stable. We now demonstrate that conditions(i) and (ii) of Theorem 2 are satisfied without any additional restrictions upon feedback functional. By substituting (39b) and (39d) in (39a) and replacing $z(t)$ by the test function $v(t)$, we get

$$
\begin{aligned}
\delta K_{f}[v, t]= & A_{1} v(t) \\
& +\int_{-\infty}^{t} d u v(u)\left(A_{2} e^{-\lambda_{x}(t-u)}\right. \\
& \left.+A_{3} e^{-\lambda(t-u)} A_{4} e^{-\lambda_{1}(t-u)}\right) \\
& +A_{5} \int_{-\infty}^{t} d u v^{2}(u) e^{-\lambda_{x}(t-u)}
\end{aligned}
$$

Also, by differentiating $\delta K_{f}[v, t]$ with respect to $t$, we obtain

$$
\begin{aligned}
\delta \dot{K}_{f}[v, t]= & A_{1} \dot{v}(t)+v(t)\left[A_{2}+A_{3}+A_{4}+A_{5} v(t)\right] \\
& -\int_{-\infty}^{t} d u v(u)\left(A_{2} \lambda_{x} e^{-\lambda_{x}(t-u)}\right. \\
& \left.+A_{3} \lambda e^{-\lambda(t-u)}+A_{4} \lambda_{1} e^{-\lambda_{1}(t-u)}\right) \\
& -A_{5} \lambda_{x} \int_{-\infty}^{t} d u v^{2}(u) e^{-\lambda_{x}(t-u)}, \quad t>0 \\
& \leq\left|A_{1}\right| M_{1}+2\left|A_{2}+A_{3}+A_{4}\right| M_{2} \\
& +2\left|A_{5}\right| M_{2}^{2}, \quad t>0,
\end{aligned}
$$

where $M_{1}, M_{2}$ are upper bounds of $\dot{v}(t)$ and $v(t)$. Hence, $\dot{\delta} K_{f}[v, t]$ is bounded, and $\delta K_{f}[v, t]$ is uniformly continuous for $t>0$. 
For condition (ii) of Theorem 2, we note that $\int_{0}^{\infty} d u \omega(t-u) A_{j} e^{-\alpha u}, j=2,3,4, \quad \alpha=\lambda_{x}, \lambda, \lambda_{1}$, and

$$
\int_{0}^{\infty} d u \omega^{2}(t-u) A_{5} e^{-\lambda_{x} u}
$$

behave as $\left(A_{j} / \alpha\right) \omega(t)$ and $\left(A_{5} / \lambda_{x}\right) \omega^{2}(t)$ as $t \rightarrow \infty$. This can be shown by following a similar procedure as that used in the relations (33)-(38). Hence,

implies that

$$
\lim _{t \rightarrow \infty} \delta K_{f}[\omega, t]=0
$$

$\lim _{t \rightarrow \infty} \omega(t)\left(A_{1}+\frac{A_{2}}{\lambda_{x}}+\frac{A_{3}}{\lambda}+\frac{A_{4}}{\lambda_{1}}+\frac{A_{5}}{\lambda_{x}} \omega(t)\right)=0$.

From Eq. (44) it is obvious that as $t \rightarrow \infty, \omega(t)$ either approaches 0 or

$$
-\frac{\lambda_{x}}{A_{5}}\left(A_{1}+\frac{A_{2}}{\lambda_{x}}+\frac{A_{3}}{\lambda}+\frac{A_{4}}{\lambda_{1}}\right) .
$$

Substitution of the values of $A_{1}, \cdots, A_{5}$ and $\lambda_{1}, \lambda, \lambda_{x}$ (cf. Ref. 7) in Eq. (45) gives a constant which is always less than -1 . But this value is not permissible by definition (cf. Footnote 1). Hence,

implies that

$$
\lim _{t \rightarrow \infty} \delta K_{f}[\omega, t]=0
$$

$$
\lim _{t \rightarrow \infty} \omega(t)=0 .
$$

\section{DISCUSSION}

In this analysis, sufficient criteria for the asymptotic stability of a reactor with arbitrary feedback have been obtained. The approach that is followed is similar to Liapunov's technique. However, since we are dealing with functionals instead of functions, just finding a positive definite Liapunov function with a negative first derivative is not enough, as was pointed out by Krasovskii." We note that the most important condition on the feedback functional is condition (ii) of Theorem 1. This condition was obtained as a sufficient criterion for asymptotic stability in Ref. 5 on the basis of considerations of energy dissipation in passive networks. The other conditions in Theorems 1 and 2 are quite mild in nature and are expected to be satisfied in actual physical systems, as was demonstrated in applications of Theorems 1 and 2 in the case of a xenon-controlled reactor with flux reactivity coefficient. ${ }^{15}$

${ }^{15}$ Pasquantonia and Kappel have recently shown ${ }^{16}$ that the condition in Ref. 5 is sufficient for asymptotic stability using Hale's theorem. ${ }^{17}$

${ }^{16}$ F. Di Pasquantonia and F. Kappel, Energia Nucl. (Milan) 15, 761 (1968).

17 J. K. Hale, J. Differential Equations 1, 452 (1965).
It is instructive to consider the conditions obtained in Theorems 1 and 2 when delayed neutrons are ignored, i.e., $a_{i}$ and $h_{i}$ are identically zero. From the proof of Theorem 2 we observe that we cannot assert asymptotic stability of the reactor when $a_{i}=h_{i} \equiv 0$ because condition (ii) of Theorem 1 guarantees only the boundedness of the solutions. It is also interesting to compare Theorem 1 with a criterion obtained by Corduneanu ${ }^{18}$ which reads as follows:

The integral equation

$$
\sigma(t)=f(t)+\int_{0}^{t} l(t-z) \varphi[\sigma(z)] d z
$$

häs at least one solution $\sigma(t)$, defined for $t \geq 0$, which satisfies

$$
\lim _{t \rightarrow \infty} \sigma(t)=0,
$$

if $f(t), l(t)$, and $\varphi(\sigma)$ are real functions satisfying the conditions:

(i) $f(t)$ is defined for $t \geq 0$ and $\vec{f}(t), \vec{f}(t) \in L_{1}(0, \infty)$; (ii)

$$
l(t)=j(t)-\rho,
$$

where $\rho>0$, and $j(t)$ is defined for $t \geq 0$ and

$$
j(t), \frac{d}{d t} j \in L_{1}(0, \infty) \cap L_{2}(0, \infty) ;
$$

(iii) $\varphi(\sigma)$ is continuous for all real $\sigma$ and satisfies

$$
\sigma \varphi(\sigma)>0 \quad(\sigma \neq 0) ;
$$

(iv) There exists a $q \geq 0$ such that

where

$$
R_{e}[(1+i \omega q) L(i \omega)] \leq 0 \quad(\omega \neq 0)
$$

$$
L(i \omega)=\int_{0}^{\infty} j(t) e^{-i \omega t} d t-\frac{\rho}{i \omega} .
$$

Equation (46) can be reduced to the point reactor kinetic equation without delayed neutrons and with a linear feedback functional [cf. Eq. (1)]

$$
\dot{z}=(1+z) \int_{-\infty}^{t} G(t-u) z(u) d u
$$

by defining

$$
\begin{aligned}
\sigma(t)= & \ln (1+z), \\
\varphi(\sigma)= & e^{\sigma}-1, \\
f(t)= & \int_{-\infty}^{0} d u G(t-u) z(u), \\
& \frac{d j}{d t}=G(t),
\end{aligned}
$$

${ }^{18}$ M. C. Corduneanu, C. R. Acad. Sci. (Paris) 256, 3564 (1963). 
and

$$
l(0)=j(0)-\rho=0 .
$$

Integrating Eq. (51d) on $(t, \infty)$, we get

$$
f(t)=-\int_{t}^{\infty} G(u) d u
$$

The condition (49) thus reduces to ${ }^{19}$

$$
R_{e}[(1+i \omega q) G(i \omega) / i \omega] \leq 0 .
$$

Hence, according to this theorem, a reactor with linear feedback is asymptotically stable in the absence of delayed neutrons if a $q \geq 0$ exists such that Eq. (53) holds and $f(t)$ and $j(t)$ satisfy conditions (i) and (ii) of Corduneanu's criterion.The conditions of Theorem 1 , on the other hand, ensure only the boundedness of reactor response in the absence of delayed neutrons, although the feedback functional may be quite arbitrary and not specifically linear. If a $q \geq 0$ cannot be found such that Eq. (53) is satisfied, then Cordune-

10 The condition (ii) of Carduneanu's theorem requires $\rho>0$, which by virtue of $(51 \mathrm{e})$ implies $j(0)>0$. The latter is the condition for the existence of a finite equilibrium power level. The condition (53) alone can not guarantee asymptotic stability. anu's theorem is noncommital. This happens to be the case for a circulating fuel reactor where $G(i \omega)$ is given by ${ }^{20}$

$$
G(i \omega)=\left(\alpha \eta / \omega^{2} \theta\right)(1-i \omega \theta-\cos \omega \theta+i \sin \omega \theta) .
$$

Here $\theta$ is the fuel transit time in the core, $\alpha$ is the temperature reactivity coefficient, and $\eta$ is the heat capacity of the reactor. Application of the relation (53) reduces Eq. (54) to

$$
q(1-\cos x)+\sin x / x \geq 1, \quad x \neq 0,
$$

where we have used the fact that $\alpha<0$ and substituted $x$ for $\omega \theta$. Clearly Eq. (55) cannot be satisfied for all $x>0$ for any choice of $q$. However, application of Theorem 1 [cf. Eq. (28)] reduces Eq. (54) to

$$
1-\cos x \geq 0 \text {. }
$$

Equation (56) ascertains the boundedness of circulating fuel reactor response to perturbations belonging to physically admissible class of initial curves.

${ }^{20}$ A. Z. Akcasu and L. M. Shotkin, Nucl. Sci. Eng. 28, 72 (1967).

\title{
Bases for Irreducible Representations of the Unitary Group in the Symplectic Group Chain
}

\author{
V. Syamala Devi \\ Andhra University, Waltair, India
}

(Received 1 March 1968; Revised Manuscript Received 9 January 1969)

\begin{abstract}
A method of obtaining the highest weight polynomials of irreducible representations $(\lambda)$ of $S p_{2 n}$ occurring in a reduction of an irreducible representation $(k)$ of $U_{2 n}$ is described. The highest weight polynomials of equivalent representations $(\lambda)$ are labeled by means of parameters which occur naturally from the Littlewood's theorem to determine the branching rules for the representations of the unitary group with respect to the symplectic subgroup, when supplemented by modification rules. The results are given explicitly for $U_{4} \supset S p_{4}$ and $U_{6} \supset S p_{6}$, the former being a canonical chain and the latter a noncanonical chain.
\end{abstract}

\section{INTRODUCTION}

The aim of this paper is to obtain a basis for any given irreducible representation (IR) of the unitary group $U_{2 n}$ in $2 n$ dimensions such that, with respect to that basis, the symplectic subgroup $S p_{2 n}$ is explicitly reduced into blocks. It is enough to find the highest weight vectors of the various IR's of $S p_{2 n}$ occurring in the reduction of a given IR of $U_{2 n}$ since all the other basis vectors in an irreducible representation space of $S p_{2 n}$ can be obtained by applying polynomials of the lowering generators of $S p_{2 n}$ on the highest weight vector because linearly independent highest weight vectors give rise to linearly independent spaces. (By the reduction of an IR of $U_{2 n}$ with respect to $S p_{2 n}$, we mean the reduction with respect to $S p_{2 n}$, of the representation of $S p_{2 n}$, obtained by considering the restriction to $S p_{2 n}$ of the given IR of $U_{2 n}$.) We use a theorem of Littlewood ${ }^{1}$ and the modification rules of

${ }^{1}$ D. E. Littlewood, Theory of Group Characters (Clarendon Press, Oxford, 1950), p. 295. 